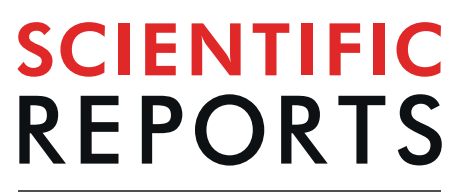

natureresearch

Check for updates

\title{
Characteristics and outcomes
} of acute-on-chronic liver failure patients with or without cirrhosis using two criteria

\author{
Xiaotian Dong ${ }^{1,5}$, Jianqin $\mathrm{He}^{2,3,5}$, Wenyi Chen ${ }^{2,3}$, Rong $\mathrm{Su}^{2}$, Yanping $\mathrm{Xu}^{2,3}$, Xinyu Sheng ${ }^{2,3}$, \\ Lanjuan $\mathrm{Li}^{2,3 凶}$ \& Hongcui $\mathrm{CaO}^{2,3,4 凶}$
}

The aim of the study was to identify the characteristics and outcomes in acute-on-chronic liver failure (ACLF) patients with or without cirrhosis using two criteria. Patients with acute deterioration of chronic hepatic disease or acute decompensation of cirrhosis were included retrospectively from April 10, 2016 to April 10, 2019. European Association for the Study of the Liver-chronic liver failure (EASL-CLIF) criterion except for consideration of cirrhosis and Chinese Group on the Study of Severe Hepatitis B (COSSH) criterion were used. Clinical features, laboratory data and survival curves were compared between the ACLF patients with and without cirrhosis. A total of 799 patients were included. Among them, 328 had COSSH and EASL ACLF, 197 had COSSH alone, and 104 had EASL alone. There were $11.6 \%$ more ACLF with COSSH criterion. Furthermore, EASL ACLF patients with non-cirrhosis vs. cirrhosis had different laboratory characteristics: ALT (423 vs. 154, $p<0.001$ ), AST (303 vs. 157, $p<0.001), \gamma-\mathrm{GT}(86$ vs. $75, p<0.01)$, and INR $(2.7$ vs. $2.6, p<0.001$ ) were significantly higher but creatinine ( 71 vs. $77, p<0.01$ ) were significantly lower; but importantly there was no statistical changes between non-cirrhosis and cirrhosis in EASL ACLF patients on 28-day $(p=0.398)$ and 90-day $(p=0.376)$ survival curves. However, 90-day $(p=0.030)$ survival curve was different between non-cirrhosis and cirrhosis in COSSH ACLF patients. COSSH ACLF score (auROC $=0.778$ or $0.792,95 \% \mathrm{Cl} 0.706-0.839$ or 0.721-0.851) displayed the better prognostic ability for EASL ACLF patients with non-cirrhosis, but CLIF-C ACLF score (auROC $=0.757$ or $0.796,95 \% \mathrm{Cl} 0.701-0.807$ or $0.743-0.843$ ) still was the best prognostic scoring system in EASL ACLF patients with cirrhosis. In conclusions, EASL definition exhibited better performance on homogeneous identification of ACLF regardless of cirrhosis or noncirrhosis. And COSSH ACLF score displayed the better prognostic ability for EASL ACLF patients without cirrhosis.

Acute-on-chronic liver failure (ACLF) is a syndrome with high 28 -day and 90-day mortality rates ${ }^{1}$ where patients with chronic hepatic disease or cirrhosis undergo acute liver deterioration. Over the last decades, various ACLF definitions have been proposed by East and West organizations. Specific definitions were provided by the Asian Pacific Association for the Study of the Liver (APASL) $)^{2,3}$ and the World Gastroenterology Organization (WGO) ${ }^{4}$ in corresponding to experts' consensus while the North American Consortium for the Study of End-Stage Liver Disease (NACSELD) Consortium ${ }^{5}$ and the European Association for the Study of the Liver-chronic liver failure (EASL-CLIF) Consortium ${ }^{6}$ defined the term based on prospective and observational study. After that, the Chinese Group on the Study of Severe Hepatitis B (COSSH) proposed a new HBV-ACLF criterion ${ }^{7}$ based on prospective study of 13 liver centers in China. Unfortunately, no definition can encompass all ACLF patients from the

\footnotetext{
${ }^{1}$ Department of Laboratory Medicine, The First Affiliated Hospital, College of Medicine, Zhejiang University, 79 Qingchun Rd, Hangzhou City, 310003, China. ${ }^{2}$ State Key Laboratory for Diagnosis and Treatment of Infectious Diseases, The First Affiliated Hospital, College of Medicine, Zhejiang University, 79 Qingchun Rd, Hangzhou City, 310003, China. ${ }^{3}$ National Clinical Research Center for Infectious Diseases, 79 Qingchun Rd, Hangzhou City, 310003, China. ${ }^{4}$ Zhejiang Provincial Key Laboratory for Diagnosis and Treatment of Aging and Physic-chemical Injury Diseases, 79 Qingchun Rd, Hangzhou City, 310003, China. ${ }^{5}$ These authors contributed equally: Xiaotian Dong and Jianqin He.®e-mail: ljli@zju.edu.cn; hccao@zju.edu.cn
} 


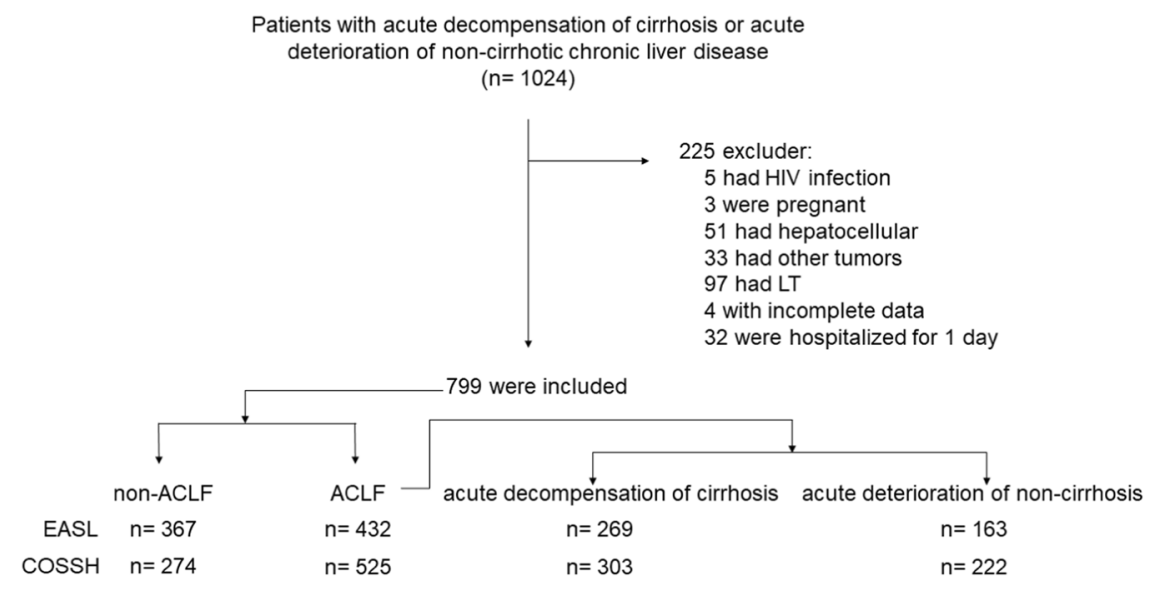

Figure 1. Distribution of patients with ACLF and non ACLF using EASL and COSSH criteria. Abbreviation: ACLF, acute-on-chronic liver failure; LT, liver transplantation; EASL, European Association for the Study of the Liver; COSSH, Chinese Group on the Study of Severe Hepatitis B.

East and West, except incomplete WGO definition ${ }^{8}$. In this study, the EASL-CLIF definition was used because of its superior abilities for defining ACLF and predicting outcome9. Moreover, the ACLF patients were also defined using the COSSH criterion, for comparability with EASL-CLIF definition.

The clinicopathological characteristics of ACLF patients with cirrhosis have been detailedly evaluated in cohorts from East and West ${ }^{6,10-12}$. However, despite the large population of non-cirrhotic ACLF patients in $\mathrm{China}^{13}$, the features and outcomes of these patients were hardly investigated. Thus, in this retrospective study, we identified clinical features of ACLF patients without cirrhosis and explored the difference between the ACLF patients with or without cirrhosis through two criteria.

\section{Patients and Methods}

Patients. Patients (Age $>18$ years) with acute decompensation (encephalopathy, ascites, upper gastrointestinal [GI] hemorrhage or bacterial infection) of cirrhosis or severe liver injury (total bilirubin [TB] $\geq 5 \mathrm{mg} / \mathrm{dL}$ and international normalized ratio $[\mathrm{INR}] \geq 1.5$ ) of non-cirrhotic chronic liver disease ${ }^{7}$ between April 10, 2016 and April 10, 2019 in the First Affiliated Hospital, Zhejiang University were screened. Cirrhosis was identified according to the results of liver biopsy, endoscopic signs of portal hypertension, previous decompensation evidence, radiological liver nodularity image and laboratory data ${ }^{12}$. Hepatic encephalopathy (HE) was graded according to the West Haven criteria ${ }^{14}$. Ascites was detected by ultrasonography ${ }^{15}$. Bacterial infection was diagnosed as previously described ${ }^{12}$. ACLF was diagnosed by EASL-CLIF definition based on CLIF-SOFA score ${ }^{6}$, and COSSH criteria ${ }^{7}$.

Patients were excluded when (1) hospitalized for only 1 day; (2) were pregnant; (3) had Acquired Immune Deficiency Syndrome; (4) had hepatocellular carcinoma; (5) had other tumors; (6) received a liver transplant; (7) had incomplete laboratory data. The study complied the Declaration of Helsinki. All experimental protocols were approved by the Ethics Committee on Clinical Research of the First Affiliated Hospital, Zhejiang University and were carried out in accordance with the approved guidelines. Informed written consent was waived due to its retrospective nature.

Data gathering. The subsequent information was gathered: general clinical records (age, sex, blood pressure, etiology, cirrhosis or non-cirrhosis), complications (HE, ascites, upper GI hemorrhage or bacterial infection), laboratory parameters and survival data. The whole data were gathered when ACLF occurried on clinical presentation or in time of hospitalization. Survival data were collected according to the medical records and outpatient information.

Study design. The clinical characteristics, laboratory data as well as mortality were contrasted using two criteria between (i) ACLF and non ACLF patients in all enrolled patients, (ii) cirrhotic ACLF patients and non-cirrhotic ACLF patients.

Statistical analysis. Categorical variables were compared by chi-square test and expressed as frequencies and percentages. Continuous variables were compared by Student's t test or Mann-Whitney U test and presented as median (IQR). Survival curves were assessed through Log-rank test. The area under the receiver operating curve (auROC) of different prognostic scoring systems, including COSSH ACLF score (COSSH ACLFs) ${ }^{7}$, CLIF Consortium ACLF score (CLIF-C ACLFs) ${ }^{16}$, CLIF-sequential organ failure assessment (CLIF-SOFA) score $^{6}$, Model for End-Stage Liver Disease (MELD) ${ }^{17}$, MELD-sodium (MELD-Na) ${ }^{18}$, and the integrated MELD (iMELD) $)^{19}$, were computed and evaluated through $Z$ test (Delong's method). Statistical analyses were accomplished by SPSS (version 21.0; IBM Corp., Armonk, NY, USA), GraphPad Prism (version 7; GraphPad Software Inc., San Diego, CA), and MedCalc software (MedCalc Software, Belgium). 


\begin{tabular}{|c|c|c|c|c|c|}
\hline \multirow[b]{2}{*}{ Characteristic } & \multicolumn{2}{|l|}{ EASL } & \multicolumn{2}{|l|}{ COSSH } & \multirow[b]{2}{*}{$p$ value } \\
\hline & $\begin{array}{l}\text { Non-ACLF } \\
(\mathrm{n}=367)\end{array}$ & $\operatorname{ACLF}(n=432)$ & $\begin{array}{l}\text { Non-ACLF } \\
(\mathrm{n}=274)\end{array}$ & $\operatorname{ACLF}(n=525)$ & \\
\hline Age (years) & $48.0(19.0)$ & $51.0(19.0)^{*}$ & $49.5(21.0)$ & $50.0(18.0)$ & 0.092 \\
\hline Male, no. (\%) & $309(84.2)$ & $338(78.2)^{*}$ & $214(78.1)$ & $433(82.5)$ & 0.099 \\
\hline \multicolumn{6}{|l|}{ Aetiology } \\
\hline HBV, no. (\%) & 328 (89.4) & $351(81.2)^{*}$ & $229(83.6)$ & $450(85.7)$ & 0.063 \\
\hline Alcohol, no. (\%) & $17(4.6)$ & $26(6.0)$ & $22(8.0)$ & $21(4.0)^{\dagger}$ & 0.150 \\
\hline $\begin{array}{l}\text { HBV + Alcohol, } \\
\text { no. (\%) }\end{array}$ & $4(1.1)$ & $16(3.7)^{*}$ & $2(0.7)$ & $18(3.4)^{\dagger}$ & 0.819 \\
\hline Others, no. (\%) & $18(4.9)$ & $39(9.0) *$ & $21(7.7)$ & $36(6.9)$ & 0.214 \\
\hline \multicolumn{6}{|l|}{ Complications } \\
\hline Ascites, no. (\%) & $281(76.6)$ & 364 (84.3)* & 209 (76.3) & $436(83.0)^{\dagger}$ & 0.614 \\
\hline $\begin{array}{l}\text { GI hemorrhage, } \\
\text { no. (\%) }\end{array}$ & $18(4.9)$ & $60(13.9) *$ & 29 (10.6) & $49(9.3)$ & $0.027^{\S}$ \\
\hline $\begin{array}{l}\text { Bacterial infection, } \\
\text { no. (\%) }\end{array}$ & 47 (12.8) & $84(19.4)^{*}$ & 47 (17.2) & $84(16.0)$ & 0.163 \\
\hline \multicolumn{6}{|l|}{ Laboratory data } \\
\hline Albumin, g/L & $31.3(6.1)$ & $30.8(6.1)$ & $30.8(6.8)$ & $31.1(5.8)$ & 0.237 \\
\hline ALT, U/L & $191.0(420.0)$ & $230.0(533.0)$ & $175.0(519.5)$ & $229.0(482.5)^{\dagger}$ & 0.897 \\
\hline AST, U/L & $144.0(288.5)$ & $190.0(365.0)^{*}$ & $146.0(374.8)$ & $173.0(302.5)$ & 0.364 \\
\hline ALP, U/L & $131.0(46.0)$ & $134.0(56.0)$ & $125.0(53.5)$ & $136.0(50.5)^{\dagger}$ & 0.334 \\
\hline $\mathrm{TB}, \mu \mathrm{mol} / \mathrm{L}$ & $258.0(200.8)$ & $372.0(219.2)^{*}$ & $161.8(80.5)$ & $358.0(157.8)^{\dagger}$ & 0.177 \\
\hline$\gamma$-GT, U/L & $94.0(83.0)$ & $78.0(74.0)^{*}$ & $101.5(88.5)$ & $78.0(74.0)^{\dagger}$ & 0.945 \\
\hline Creatinine, $\mu \mathrm{mol} / \mathrm{L}$ & $65.0(19.0)$ & $75.0(49.0)^{*}$ & $66.5(24.0)$ & $67.0(23.0)$ & $<0.001^{5}$ \\
\hline Sodium, mmol/L & $138.0(4.0)$ & $137.0(6.0)^{*}$ & $138.0(5.3)$ & $137.0(4.0)^{\dagger}$ & 0.858 \\
\hline INR & $1.8(0.4)$ & $2.6(1.0)^{*}$ & $1.8(0.7)$ & $2.1(0.8)^{\dagger}$ & $<0.001^{\varsigma}$ \\
\hline WBC, $10^{9} / \mathrm{L}$ & $6.0(3.4)$ & $7.0(4.6)^{*}$ & $6.0(3.5)$ & $6.8(4.2)^{\dagger}$ & 0.282 \\
\hline Hemoglobin, g/L & $126.0(26.5)$ & $121.0(31.0)$ & $121.5(33.0)$ & $125.0(27.0)^{\dagger}$ & 0.071 \\
\hline Hematocrit, \% & $35.8(8.3)$ & $34.8(9.0)^{*}$ & $35.1(10.1)$ & $35.4(8.5)$ & 0.221 \\
\hline Platelet, $10^{9} / \mathrm{L}$ & $101.0(72.5)$ & $99.0(74.0)$ & $100.5(81.3)$ & $100.0(70.0)$ & 0.622 \\
\hline $\begin{array}{l}\mathrm{C} \text { reactive protein, } \\
\mathrm{mg} / \mathrm{L}\end{array}$ & $11.9(10.5)$ & $12.0(12.5)$ & $11.9(15.4)$ & $12.0(10.5)$ & 0.921 \\
\hline $\begin{array}{l}\text { Alpha fetoprotein, } \\
\mu \mathrm{g} / \mathrm{L}\end{array}$ & $89.7(256.5)$ & $42.4(131.8)^{*}$ & $38.1(224.1)$ & $76.5(211.4)^{\dagger}$ & $<0.001^{\S}$ \\
\hline Ferritin, $\mu \mathrm{g} / \mathrm{L}$ & $1779.1(2411.7)$ & $2653.9(3799.8)^{*}$ & $1813.3(2745.0)$ & $2574.9(3473.9)^{\dagger}$ & 0.985 \\
\hline \multicolumn{6}{|l|}{ Organ failure } \\
\hline Liver, no. (\%) & $268(73.0)$ & $411(95.1)^{*}$ & $154(56.2)$ & $525(100.0)^{\dagger}$ & $<0.001^{\varsigma}$ \\
\hline Kidney, no. (\%) & $0(0.0)$ & $90(20.8) *$ & $19(6.9)$ & $71(13.5)^{\dagger}$ & $0.003^{\S}$ \\
\hline Cerebral, no. (\%) & $2(0.5)$ & $100(23.1)^{*}$ & $22(8.0)$ & $80(15.2)^{\dagger}$ & $0.002^{\S}$ \\
\hline $\begin{array}{l}\text { Coagulation, no. } \\
(\%)\end{array}$ & $11(3.0)$ & $315(72.9)^{*}$ & 78 (28.5) & $248(47.2)^{\dagger}$ & $<0.001^{\S}$ \\
\hline $\begin{array}{l}\text { Circulation, no. } \\
(\%)\end{array}$ & $1(0.3)$ & $72(16.7)^{*}$ & $19(6.9)$ & $54(10.3)$ & $0.004^{\S}$ \\
\hline Lung, no. (\%) & $0(0.0)$ & $62(14.4) *$ & $15(5.5)$ & $47(8.9)$ & $0.009^{\lessgtr}$ \\
\hline $\begin{array}{l}\text { Hepatic } \\
\text { encephalopathy } \\
\text { grade I or II }\end{array}$ & $12(3.3)$ & $143(33.1)^{*}$ & $31(11.3)$ & $59(11.2)$ & $<0.001 \S$ \\
\hline \multicolumn{6}{|l|}{ Severity score } \\
\hline COSSH ACLFs & $5.2(0.6)$ & $6.3(1.5)^{*}$ & $5.4(1.0)$ & $5.8(1.3)^{\dagger}$ & $<0.001^{\S}$ \\
\hline CLIF-C ACLFs & $37.7(8.7)$ & $49.6(15.0)^{*}$ & $37.5(12.1)$ & $44.2(13.9)^{\dagger}$ & $<0.001^{5}$ \\
\hline CLIF-SOFA & $8.0(1.0)$ & $11.0(3.0)^{*}$ & $8.0(2.0)$ & $10.0(2.0)^{\dagger}$ & $<0.001^{5}$ \\
\hline MELD & $19.9(5.4)$ & $27.4(7.7)^{*}$ & $18.1(6.1)$ & $23.5(6.4)^{\dagger}$ & 0.109 \\
\hline MELD-Na & $21.4(5.4)$ & $28.4(7.7)^{*}$ & $19.6(5.7)$ & $25.2(6.0)^{\dagger}$ & 0.108 \\
\hline iMELD & $3.5(0.9)$ & $5.8(4.2)^{*}$ & $3.6(1.8)$ & $4.3(2.8)$ & $<0.001^{5}$ \\
\hline \multicolumn{6}{|c|}{ Transplant-free mortality } \\
\hline 28-day, no. (\%) & $37(10.1)$ & $217(50.2)^{*}$ & $59(21.5)$ & $195(37.1)^{\dagger}$ & $<0.001^{\S}$ \\
\hline 90-day, no. (\%) & $44(12.0)$ & $247(57.2)^{*}$ & $68(24.8)$ & $223(42.5)^{\dagger}$ & $<0.001^{5}$ \\
\hline Cirrhosis & $210(57.2)$ & $269(62.3)$ & $176(64.2)$ & $303(57.7)$ & 0.153 \\
\hline
\end{tabular}

Table 1. Characteristics of patients with ACLF and non-ACLF. ${ }^{\circledR} \mathrm{p}<0.05$, ACLF patients, EASL-ACLF vs. COSSH-ACLF. ${ }^{*} \mathrm{p}<0.05$, patients with EASL definition, Non-ACLF vs. ACLF. ${ }^{\dagger} \mathrm{p}<0.05$, patients with COSSH definition, Non-ACLF vs. ACLF. 
EASL

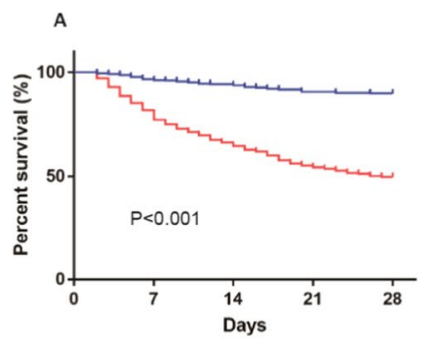

B

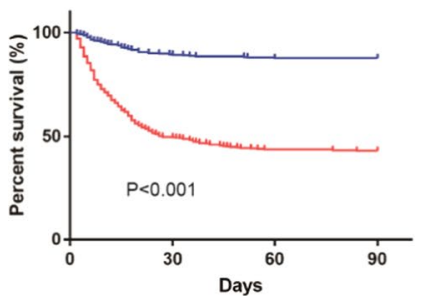

$\operatorname{cossh}$
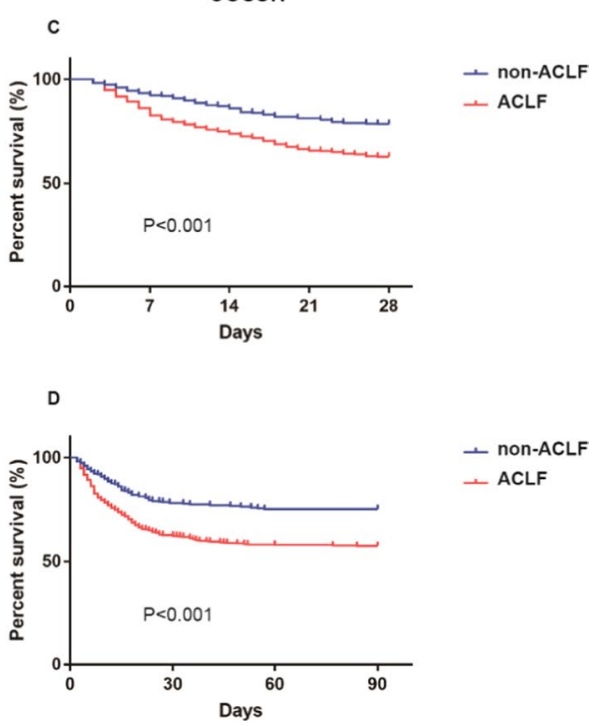

+ non-ACLF

- ACLF

Figure 2. 28-day and 90-day survival curves of ACLF and non-ACLF patients using EASL and COSSH criteria and Log-rank test were used to compare two groups. Abbreviation: ACLF, acute-on-chronic liver failure; EASL, European Association for the Study of the Liver; COSSH, Chinese Group on the Study of Severe Hepatitis B.

\section{Results}

Different groups of patients. A total of 799 patients who developed acute decompensation (AD) of cirrhosis and acute liver deterioration (ALD) of non-cirrhotic chronic hepatic disease were included after excluding 225 patients (Fig. 1). Among them, 328 developed COSSH- and EASL- defined ACLF, 197 developed COSSH-defined ACLF (COSSH ACLF) alone, and 104 developed EASL-defined ACLF (EASL ACLF) alone. The incidence rate for COSSH ACLF and EASL ACLF was 65.7\% (525/799) and 54.1\% (432/799), respectively. There were $11.6 \%$ more of ACLF cases when defined by COSSH criteria.

EASL ACLF was more severe and with higher short time mortality than COSSH ACLF. The detailed comparision of the characteristics between EASL ACLF and COSSH ACLF patients was displayed in Table 1. ACLF and non-ACLF patients were mainly HBV carriers, and ACLF patients were older than non-ACLF patients. In addition, ADs occurred more frequently in ACLF patients although there was no discrepancy between ACLF and non- ACLF patients in the prevalence of cirrhosis. Compared to non ACLF patients, the levels of TB, INR, WBC count and ferritin were significantly higher while $\gamma$-GT and serum sodium were significantly lower in EASL ACLF and COSSH ACLF patients. Liver and coagulation failure were most commonly seen in ACLF patients defined by two criteria. Six prognostic scoring systems indicated a worse outcome for EASL ACLF and COSSH ACLF patients than non-ACLF patients, in accordance with 28-day and 90-day survival curves (Table 1, Fig. 2).

Moreover, compared to COSSH ACLF patients, the levels of Creatinine and INR were significantly higher while alpha fetoprotein was significantly lower in EASL ACLF. Organ failures, except for liver failure, occured more frequently in EASL ACLF compared to COSSH ACLF. Four prognostic scoring systems indicated a worse outcome for EASL ACLF than COSSH ACLF patients, consistent with 28-day and 90-day survival rates (Table 1).

EASL ACLF patients with cirrhosis and non-cirrhosis had a more consistent outcome. The detail of characteristics between ACLF patients with cirrhosis and non-cirrhosis was compared in Table 2. EASL ACLF and COSSH ACLF patients with non-cirrhosis were younger and had more HBV infection than cirrhotic EASL ACLF and COSSH ACLF patients. But ADs were happened more commonly in both EASL ACLF and COSSH ACLF patients with cirrhosis. The measures of albumin, ALT, AST, $\gamma$-GT, serum sodium, WBC count, hemoglobin, hematocrit, platelet count, alpha fetoprotein and ferritin were significantly higher but $\mathrm{c}$ reactive protein were significantly lower in EASL ACLF and COSSH ACLF patients with non-cirrhosis, compared with ACLF patients with cirrhosis. In addition, lower occurrence of kidney failure was observed in EASL ACLF and COSSH ACLF patients with non-cirrhosis, compared with ACLF patients with cirrhosis. Six prognostic scoring systems predicted no statistical difference in outcomes between EASL ACLF patient with cirrhosis and non-cirrhosis. And there was also no statistical alteration between EASL ACLF patient with cirrhosis and non-cirrhosis on 28-day and 90-day survival curves (Fig. 3). However, COSSH ACLF score, CLIF-C ACLF score and iMELD score indicated a worse outcome for COSSH ACLF patients with cirrhosis than non-cirrhosis, and 90-day survival curves were consistent with that (Table 2, Fig. 3).

CLIF-C ALCF score was better in predicting ACLF patients with cirrhosis short time mortality, but COSSH ACLF score was better for ACLF patients with non-cirrhosis. In all EASL ACLF patients and EASL ACLF patients with non-cirrhosis, COSSH ACLFs possessed the best predictive value of 28-day and 90-day mortality among six prognostic scoring systems (Table 3). And CLIF-C ACLFs, CLIF-SOFA and iMELD scores also had good predictive value in those patients. However, CLIF-C ALCFs still was the best 


\begin{tabular}{|c|c|c|c|c|c|}
\hline \multirow[b]{2}{*}{ Characteristic } & \multicolumn{2}{|l|}{ EASL ACLF } & \multicolumn{2}{|l|}{ COSSH ACLF } & \multirow[b]{2}{*}{$p$ value } \\
\hline & $\begin{array}{l}\text { Cirrhosis } \\
(n=269)\end{array}$ & $\begin{array}{l}\text { Non-cirrhosis } \\
(\mathrm{n}=163)\end{array}$ & $\begin{array}{l}\text { Cirrhosis } \\
(\mathbf{n}=303)\end{array}$ & $\begin{array}{l}\text { Non-cirrhosis } \\
(\mathbf{n}=\mathbf{2 2 2})\end{array}$ & \\
\hline Age (years) & $53(17)$ & $46(18)^{*}$ & $53(16)$ & $45(19)^{\dagger}$ & 0.498 \\
\hline Male, no. (\%) & $204(75.8)$ & $134(82.2)$ & $238(78.5)$ & $195(87.8)^{\dagger}$ & 0.122 \\
\hline \multicolumn{6}{|l|}{ Aetiology } \\
\hline HBV, no. (\%) & $197(73.2)$ & $154(94.5)^{*}$ & $234(77.2)$ & $216(97.3)^{\dagger}$ & 0.158 \\
\hline Alcohol, no. (\%) & $24(8.9)$ & $2(1.2)^{*}$ & $20(6.6)$ & $1(0.5)^{\dagger}$ & 0.576 \\
\hline $\begin{array}{l}\text { HBV + Alcohol, } \\
\text { no. (\%) }\end{array}$ & $14(5.2)$ & $2(1.2)^{*}$ & $16(5.3)$ & $2(0.9)^{\dagger}$ & 1.000 \\
\hline Others, no. (\%) & $34(12.6)$ & $5(3.1)^{*}$ & $33(10.9)$ & $3(1.4)^{\dagger}$ & 0.291 \\
\hline \multicolumn{6}{|l|}{ Complications } \\
\hline Ascites, no. (\%) & 255 (94.8) & $109(66.9)^{*}$ & $289(95.4)$ & $147(66.2)^{\dagger}$ & 0.893 \\
\hline $\begin{array}{l}\text { GI hemorrhage, } \\
\text { no. (\%) }\end{array}$ & $49(18.2)$ & $11(6.7)^{*}$ & 39 (12.9) & $10(4.5)^{\dagger}$ & 0.338 \\
\hline $\begin{array}{l}\text { Bacterial infection, } \\
\text { no. (\%) }\end{array}$ & 67 (24.9) & $17(10.4)^{*}$ & $67(22.1)$ & $17(7.7)^{\dagger}$ & 0.344 \\
\hline \multicolumn{6}{|l|}{ Laboratory data } \\
\hline Albumin, $\mathrm{g} / \mathrm{L}$ & $30.2(5.5)$ & $31.9(5.7)^{*}$ & $30.9(5.7)$ & $31.7(5.6)^{\dagger}$ & 0.678 \\
\hline ALT, U/L & $154.0(356.0)$ & $423.0(692.0)^{*}$ & $149.0(328.0)$ & $401.5(608.3)^{\dagger}$ & 0.365 \\
\hline AST, U/L & $157.0(253.0)$ & $303.0(404.0)^{*}$ & $148.0(213.0)$ & $234.5(362.3)^{\dagger}$ & 0.193 \\
\hline ALP, U/L & $131.5(57.0)$ & $140.0(55.0)$ & $135.0(50.0)$ & $137.0(50.3)$ & 0.829 \\
\hline $\mathrm{TB}, \mu \mathrm{mol} / \mathrm{L}$ & $371.5(239.2)$ & $375.0(189.0)$ & $368.0(167.0)$ & $342.1(145.5)^{\dagger}$ & $0.025^{\S}$ \\
\hline$\gamma$-GT, U/L & $75.0(67.8)$ & $86.0(74.0)^{*}$ & $74.0(57.0)$ & $87.5(78.5)^{\dagger}$ & 0.772 \\
\hline Creatinine, $\mu \mathrm{mol} / \mathrm{L}$ & $77.0(60.5)$ & $71.0(43.0)^{*}$ & $67.0(25.0)^{\#}$ & $64.0(19.3)$ & $<0.001^{s}$ \\
\hline Sodium, mmol/L & $137.0(6.0)$ & $138.0(4.0)^{*}$ & $137.0(5.0)$ & $138.0(4.0)^{\dagger}$ & 0.693 \\
\hline INR & $2.6(1.0)$ & $2.7(1.0)^{*}$ & $2.1(0.7)^{\#}$ & $2.1(0.9)$ & $<0.001^{s}$ \\
\hline WBC, $10^{9} / \mathrm{L}$ & $6.8(4.2)$ & $7.4(4.8)^{*}$ & $6.5(4.1)$ & $7.1(3.8)^{\dagger}$ & 0.167 \\
\hline Hemoglobin, g/L & $116.0(29.0)$ & $132.0(28.0)^{*}$ & $119.0(26.0)$ & $133.0(22.3)^{\dagger}$ & 0.346 \\
\hline Hematocrit, \% & $33.5(8.1)$ & $38.0(8.5)^{*}$ & $34.1(7.1)$ & $38.0(7.1)^{\dagger}$ & 0.837 \\
\hline Platelet, $10^{9} / \mathrm{L}$ & $84.0(69.0)$ & $119.0(73.0)^{*}$ & $86.0(68.0)$ & $118.5(70.5)^{\dagger}$ & 0.694 \\
\hline $\begin{array}{l}\text { C reactive protein, } \\
\mathrm{mg} / \mathrm{L}\end{array}$ & $13.8(13.9)$ & $10.2(8.1)^{*}$ & $13.2(11.2)$ & $10.7(8.8)^{\dagger}$ & 0.290 \\
\hline $\begin{array}{l}\text { Alpha fetoprotein, } \\
\mu \mathrm{g} / \mathrm{L}\end{array}$ & $37.7(112.8)$ & $53.9(189.3)^{*}$ & $59.9(151.1)^{\#}$ & $109.3(235.5)^{\dagger}$ & $0.001^{\S}$ \\
\hline Ferritin, $\mu \mathrm{g} / \mathrm{L}$ & $2098.3(3288.8)$ & $3404.0(3934.0)^{*}$ & $2115.7(3036.2)$ & $3105.4(3918.9)^{\dagger}$ & 0.328 \\
\hline \multicolumn{6}{|l|}{ Organ faliure } \\
\hline Liver, no. (\%) & $253(94.1)$ & $158(96.9)$ & $303(100.0)^{\#}$ & $222(100.0)$ & $0.013^{\varsigma}$ \\
\hline Kidney, no. (\%) & $66(24.5)$ & $24(14.7)^{*}$ & $50(16.5)^{\#}$ & $21(9.5)^{\dagger}$ & 0.112 \\
\hline Cerebral, no. (\%) & $57(21.2)$ & $43(26.4)$ & $44(14.5)^{\#}$ & $36(16.2)$ & $0.015^{\S}$ \\
\hline $\begin{array}{l}\text { Coagulation, no. } \\
\text { (\%) }\end{array}$ & $186(69.1)$ & $129(79.1)^{*}$ & $145(47.9)^{\#}$ & $103(46.4)$ & $<0.001^{s}$ \\
\hline $\begin{array}{l}\text { Circulation, no. } \\
(\%)\end{array}$ & 47 (17.5) & $25(15.3)$ & $33(10.9)^{\#}$ & $21(9.5)$ & 0.079 \\
\hline Lung, no. (\%) & $36(13.4)$ & $26(16.0)$ & $25(8.3)^{\#}$ & $22(9.9)$ & $0.036^{\S}$ \\
\hline $\begin{array}{l}\text { Hepatic } \\
\text { encephalopathy } \\
\text { grade I or II }\end{array}$ & $94(34.9)$ & $49(30.1)$ & $67(22.1)^{\#}$ & $61(27.5)$ & 0.579 \\
\hline \multicolumn{6}{|l|}{ Severity score } \\
\hline COSSH ACLFs & $6.3(1.4)$ & $6.2(1.7)$ & $5.9(1.2)^{\#}$ & $5.7(1.4)^{\dagger}$ & $<0.001^{s}$ \\
\hline CLIF-C ACLFs & $50.9(14.4)$ & $48(16.7)$ & $45.4(13.5)^{\#}$ & $42.3(13.2)^{\dagger}$ & $0.002^{\S}$ \\
\hline CLIF-SOFA & $11.0(3.0)$ & $11.0(3.0)$ & $10.0(2.0)^{\#}$ & $9.0(2.0)$ & $<0.001^{\S}$ \\
\hline MELD & $27.3(8.4)$ & $27.7(6.9)$ & $23.5(6.6)$ & $23.5(5.9)$ & $0.019^{\S}$ \\
\hline MELD-Na & $28.3(7.9)$ & $28.6(6.9)$ & $25.3(6.1)$ & $25.0(6.2)$ & $0.022^{\varsigma}$ \\
\hline iMELD & $5.8(4.1)$ & $5.9(4.7)$ & $4.4(2.5)^{\#}$ & $4.2(3.4)^{\dagger}$ & $<0.001^{\mathrm{s}}$ \\
\hline \multicolumn{6}{|c|}{ Transplant-free mortality } \\
\hline 28-day, no. (\%) & $139(51.7)$ & 78 (47.9) & $122(40.3)^{\#}$ & $73(32.9)$ & $0.003^{\varsigma}$ \\
\hline 90-day, no. (\%) & $166(61.7)$ & $81(49.7)^{*}$ & $144(47.5)^{\#}$ & 79 (35.6) & $0.006^{\S}$ \\
\hline
\end{tabular}

Table 2. Characteristics of ACLF patients with cirrhosis and non-cirrhosis. ${ }^{\S} \mathrm{p}<0.05$, ACLF patients with Noncirrhosis, EASL ACLF with Non-cirrhosis vs. COSSH ACLF with Non-cirrhosis. ${ }^{*} \mathrm{p}<0.05$, ACLF patients with Cirrhosis, EASL ACLF with Cirrhosis vs. COSSH ACLF with Cirrhosis. *p $<0.05$, ACLF patients with EASL definition, Cirrhosis vs. Non-cirrhosis. ${ }^{\dagger} p<0.05$, ACLF patients with COSSH definition, Cirrhosis vs. Noncirrhosis. 
EASL
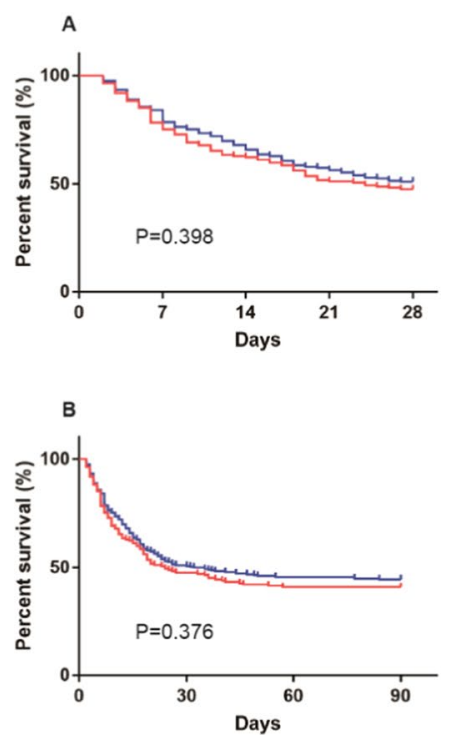

$\operatorname{cossh}$

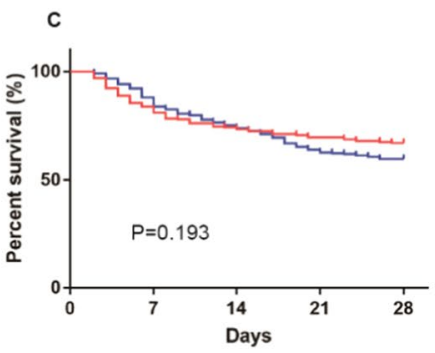

$$
+ \text { Non-cirrhosis }
$$$$
+ \text { Cirrhosis }
$$

- Cirrhosis

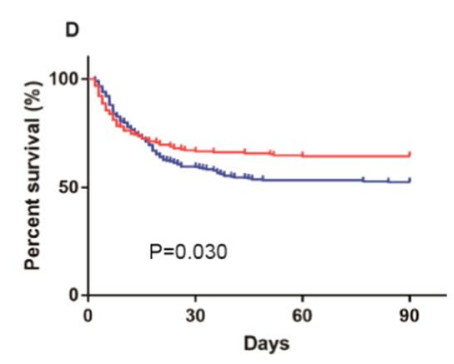

- Non-cirrhosis

- Cirrhosis

- Non-cirrhosis

+ Cirrhosis

Figure 3. 28-day and 90-day survival curves of ACLF patients with cirrhosis and non-cirrhosis using EASL and COSSH criteria and Log-rank test were used to compare two groups. Abbreviation: ACLF, acute-on-chronic liver failure; EASL, European Association for the Study of the Liver; COSSH, Chinese Group on the Study of Severe Hepatitis B.

\begin{tabular}{|c|c|c|c|c|c|c|c|c|}
\hline & \multicolumn{4}{|l|}{ 28-day } & \multicolumn{4}{|l|}{ 90-day } \\
\hline & auROC & $95 \%$ CI & \begin{tabular}{|l|}
$Z$ \\
value
\end{tabular} & $p$ value & auROC & $95 \% \mathrm{CI}$ & \begin{tabular}{|l|}
$Z$ \\
value
\end{tabular} & $p$ value \\
\hline \multicolumn{9}{|c|}{ All ACLF patients } \\
\hline COSSH ACLFs & 0.778 & $0.706-0.839$ & & & 0.792 & $0.721-0.851$ & & \\
\hline CLIF-C ACLFs & 0.754 & $0.680-0.818$ & 0.891 & 0.373 & 0.765 & $0.692-0.828$ & 0.983 & 0.326 \\
\hline CLIF-SOFA & 0.765 & $0.692-0.828$ & 0.479 & 0.632 & 0.778 & $0.706-0.839$ & 0.518 & 0.604 \\
\hline MELD & 0.605 & $0.525-0.680$ & 3.676 & $<0.001$ & 0.602 & $0.523-0.678$ & 4.088 & $<0.001$ \\
\hline MELD-Na & 0.620 & $0.541-0.695$ & 3.474 & $<0.001$ & 0.616 & $0.537-0.691$ & 3.926 & $<0.001$ \\
\hline iMELD & 0.761 & $0.688-0.824$ & 0.521 & 0.602 & 0.766 & $0.693-0.828$ & 0.803 & 0.422 \\
\hline \multicolumn{9}{|c|}{ ACLF patients with cirrhosis } \\
\hline COSSH ACLFs & 0.726 & $0.669-0.779$ & & & 0.767 & $0.712-0.816$ & & \\
\hline CLIF-C ACLFs & 0.757 & $0.701-0.807$ & 1.304 & 0.192 & 0.796 & $0.743-0.843$ & 1.213 & 0.225 \\
\hline CLIF-SOFA & 0.740 & $0.683-0.791$ & 0.550 & 0.582 & 0.787 & $0.733-0.834$ & 0.782 & 0.434 \\
\hline MELD & 0.612 & $0.551-0.671$ & 2.878 & 0.004 & 0.581 & $0.520-0.641$ & 4.659 & $<0.001$ \\
\hline MELD-Na & 0.624 & $0.563-0.682$ & 2.545 & 0.011 & 0.590 & $0.528-0.649$ & 4.357 & $<0.001$ \\
\hline iMELD & 0.753 & $0.697-0.803$ & 0.881 & 0.378 & 0.748 & $0.692-0.799$ & 0.619 & 0.536 \\
\hline \multicolumn{9}{|c|}{ ACLF patients without cirrhosis } \\
\hline COSSH ACLFs & 0.778 & $0.706-0.839$ & & & 0.792 & $0.721-0.851$ & & \\
\hline CLIF-C ACLFs & 0.754 & $0.680-0.818$ & 0.891 & 0.373 & 0.765 & $0.692-0.828$ & 0.983 & 0.326 \\
\hline CLIF-SOFA & 0.765 & $0.692-0.828$ & 0.479 & 0.632 & 0.778 & $0.706-0.839$ & 0.518 & 0.604 \\
\hline MELD & 0.605 & $0.525-0.680$ & 3.676 & $<0.001$ & 0.602 & $0.523-0.678$ & 4.088 & $<0.001$ \\
\hline MELD-Na & 0.620 & $0.541-0.695$ & 3.474 & $<0.001$ & 0.616 & $0.537-0.691$ & 3.926 & $<0.001$ \\
\hline iMELD & 0.761 & $0.688-0.824$ & 0.521 & 0.602 & 0.766 & $0.693-0.828$ & 0.803 & 0.422 \\
\hline
\end{tabular}

Table 3. Predictive value of six prognostic scoring systems in ACLF patients. Data were compared by $Z$ test (Delong's method)

prognostic scoring system in EASL ACLF patients with cirrhosis. Furthermore, COSSH ACLFs, CLIF-SOFA and iMELD also had a well performance in prediction of these patients' outcomes.

\section{Discussion}

ACLF is a syndrome accompanied by multisystem organ failure and high 28-day and 90-day mortality. The cause of ACLF is dissimilar in the East and West. The East ACLF patients are primarily developed from the viral (hepatitis $\mathrm{B}$ or $\mathrm{C}$ ) related chronic hepatic disease (with or without cirrhosis) ${ }^{20}$. And various HBV-ACLF 
related prognostic models based on serum miRNAs or multicenter data were established ${ }^{7,21}$. In this study, we attempted to obtain the variance between the ACLF patients with and without cirrhosis using the two definitions (EASL-CLIF definition and COSSH definition), and also verified which one was more appropriate definition for ACLF patients.

Our study indicated that ACLF had similar prevalence in patients with cirrhosis and non-cirrhosis using two definitions (Fig. 1). And, coagulation failure was the most common organ failure in our ACLF patients (EASL ACLF and COSSH ACLF), except for liver failure, which was different with the CANONIC study ${ }^{6}$. In addition, ACLF patients were older and had more severe deterioration of laboratory parameters than non-ACLF patients, which paralleled the outcomes of ACLF patients. However, EASL ACLF patients had more severe kidney function and coagulation function (higher level of creatinine and INR) accompanied by higher prognostic scores and worse outcomes, compared with COSSH ACLF patients. These results indicated that COSSH definition improved the sensitivity for finding more ACLF patients (11.6\%) but also reduced some important characteristics of ACLF patients, for example supposedly worse kidney and coagulation function.

Importantly, although EASL ACLF and COSSH ACLF patients with non-cirrhosis both had distinct characteristics with ACLF patients with cirrhosis, but similar outcomes and prognostic scores of ACLF patients with cirrhosis and non-cirrhosis were observed only in EASL definition (Table 2, Fig. 3). These data indicated that COSSH ACLF patients with non-cirrhosis exhibited higher levels of ALT and AST but relatively lower level of $\mathrm{TB}$, compared with COSSH ACLF patients with cirrhosis. In addition, COSSH ACLF patients with non-cirrhosis exhibited similar level of creatinine with COSSH ACLF cirrhosis patients, but higher proportion of kidney failure was observed in COSSH ACLF cirrhosis patients. However, EASL ACLF patients with non-cirrhosis exhibited worse liver function (higher levels of ALT and AST) and coagulation function (higher level of INR) but relatively better kidney function (lower level of creatinine) than EASL ACLF patients with cirrhosis. In addition, EASL ACLF patients with non-cirrhosis were younger and exhibited higher occurrence of coagulation failure and lower occurrence of kidney failure and ADs. These results indicated our EASL ACLF patients with non-cirrhosis might also meet APASL definition (TB $\geq 5 \mathrm{mg} / \mathrm{dL}$ and INR $\geq 1.5$ complicated within 4 weeks by clinical ascites and/ or encephalopathy $)^{22}$. Actually, in 163 EASL ACLF patients with non-cirrhosis, 143 (87.7\%) developed APASL and EASL ACLF in our study. This result verified that EASL definition also had good performance on diagnosis of ACLF patients with non-cirrhosis. Importantly, EASL ACLF patients with cirrhosis and non-cirrhosis had a more consistent prognostic score and outcome. Moreover, both EASL ACLF patients with and without cirrhosis were possessed similar relatively high occurrence of liver failure. Thus, the development of ACLF patients was highly determined by the liver function and EASL definition exhibited better performance on homogeneous identification of ACLF.

ACLF patients always exhibit one or more organ failures and have high mortality rates. In our study, the short time mortality of EASL ACLF patients with and without cirrhosis are similar to other studies ${ }^{7,12}$. And there was no statistical difference between EASL ACLF patients with and without cirrhosis on 28-day and 90-day survival curves (Fig. 3). Furthermore, COSSH ACLF score $(0.741 \times \mathrm{INR}+0.523 \times \mathrm{HBV}-\mathrm{SOFA}+0.026 \times$ age $+0.003 \times$ $\mathrm{TB})^{7}$, not CLIF-C ACLF score, had the best predictive value on the 28-day and 90-day mortality in ACLF patients with non-cirrhosis. Interestingly, iMELD score, as TB, creatinine, INR, age and HE are main element in iMELD score $^{19}$, CLIF-SOFA and CLIF-C ACLF score also had well performance on predicting short time prognosis of ACLF patients with non-cirrhosis. However, CLIF-C ACLF score $(10 \times[0.33 \times$ CLIF-OFs $+0.04 \times$ age +0.63 $\times \ln$ (WBC count)-2) still was the best prognostic scoring system in EASL ACLF patients with cirrhosis, probably because age and systemic inflammation (high WBC count) were strongly associated with the worsen of ACLF patients with cirrhosis ${ }^{16,23}$.

Considering this is a single center study that potential patient selection bias may exist, multicenter prospective study was needed in the future. In summary, we identified EASL definition was better and observed the distinct characteristics but similar outcomes between EASL ACLF patients with and without cirrhosis. Moreover, COSSH ACLF score displayed the better prognostic ability for ACLF patients with non-cirrhosis, but CLIF-C ACLF score still was the best prognostic scoring system in EASL ACLF patients with cirrhosis.

Received: 28 January 2020; Accepted: 6 May 2020;

Published online: 22 May 2020

\section{References}

1. Hernaez, R., Sola, E., Moreau, R. \& Gines, P. Acute-on-chronic liver failure: an update. Gut 66, 541-552 (2017)

2. Sarin, S. K. et al. Acute-on-chronic liver failure: consensus recommendations of the Asian Pacific Association for the study of the liver (APASL). Hepatol Int 3, 269-282 (2009).

3. Sarin, S. K. et al. Acute-on-chronic liver failure: consensus recommendations of the Asian Pacific Association for the Study of the Liver (APASL) 2014. Hepatol Int 8, 453-471 (2014).

4. Jalan, R. et al. Toward an improved definition of acute-on-chronic liver failure. Gastroenterology 147, 4-10 (2014).

5. Bajaj, J. S. et al. Survival in Infection-Related Acute-on-Chronic Liver Failure Is Defined by Extrahepatic Organ Failures. Hepatology 60, 250-256 (2014).

6. Moreau, R. et al. Acute-on-Chronic Liver Failure Is a Distinct Syndrome That Develops in Patients With Acute Decompensation of Cirrhosis. Gastroenterology 144, 1426-U1189 (2013).

7. Wu, T. et al. Development of diagnostic criteria and a prognostic score for hepatitis B virus-related acute-on-chronic liver failure. Gut 67, 2181-2191 (2018).

8. Duseja, A. \& Singh, S. P. Toward a Better Definition of Acute-on-Chronic Liver Failure. J Clin Exp Hepatol 7, 262-265 (2017).

9. Dhiman, R. K., Agrawal, S., Gupta, T., Duseja, A. \& Chawla, Y. Chronic Liver Failure-Sequential Organ Failure Assessment is better than the Asia-Pacific Association for the Study of Liver criteria for defining acute-on-chronic liver failure and predicting outcome. World J Gastroenterol 20, 14934-14941 (2014).

10. Katoonizadeh, A. et al. Early features of acute-on-chronic alcoholic liver failure: a prospective cohort study. Gut 59, 1561-1569 (2010). 
11. Li, H. et al. Submassive hepatic necrosis distinguishes HBV-associated acute on chronic liver failure from cirrhotic patients with acute decompensation. J Hepatol 63, 50-59 (2015).

12. Shi, Y. et al. Acute-on-chronic liver failure precipitated by hepatic injury is distinct from that precipitated by extrahepatic insults. Hepatology 62, 232-242 (2015).

13. Gu, W. Y. et al. Acute-on-Chronic Liver Failure in China: Rationale for Developing a Patient Registry and Baseline Characteristics. Am J Epidemiol 187, 1829-1839 (2018).

14. Bajaj, J. S. Review article: the modern management of hepatic encephalopathy. Aliment Pharmacol Ther 31, 537-547 (2010).

15. European Association for the Study of the, L. EASL clinical practice guidelines on the management of ascites, spontaneous bacterial peritonitis, and hepatorenal syndrome in cirrhosis. J Hepatol 53, 397-417 (2010).

16. Jalan, R. et al. Development and validation of a prognostic score to predict mortality in patients with acute-on-chronic liver failure. J Hepatol 61, 1038-1047 (2014).

17. Wiesner, R. et al. Model for end-stage liver disease (MELD) and allocation of donor livers. Gastroenterology 124, 91-96 (2003).

18. Kim, W. R. et al. Hyponatremia and mortality among patients on the liver-transplant waiting list. N Engl J Med 359, 1018-1026 (2008).

19. Yan, H. et al. A novel integrated Model for End-Stage Liver Disease model predicts short-term prognosis of hepatitis B virus-related acute-on-chronic liver failure patients. Hepatol Res 45, 405-414 (2015).

20. Abbas, Z. \& Shazi, L. Pattern and profile of chronic liver disease in acute on chronic liver failure. Hepatol Int 9, 366-372 (2015).

21. Wen, Y. et al. Serum levels of miRNA in patients with hepatitis B virus-associated acute-on-chronic liver failure. Hepatobiliary Pancreat Dis Int 17, 126-132 (2018).

22. Sarin, S. K. et al. Acute-on-chronic liver failure: consensus recommendations of the Asian Pacific association for the study of the liver (APASL): an update. Hepatol Int 13, 353-390 (2019).

23. Claria, J. et al. Systemic Inflammation in Decompensated Cirrhosis: Characterization and Role in Acute-on-Chronic Liver Failure. Hepatology 64, 1249-1264 (2016).

\section{Acknowledgements}

This study was supported by National Science and Technology Major Project for Infectious Diseases (No. 2012ZX10002004).

\section{Author contributions}

X.D. contributed to experimental design, interpretation of data, and manuscript writing. J.H., W.C., Y.X., R.S. and X.S. contributed to interpretation of data. L.L. contributed to study supervision. H.C. conducted the conception, design and manuscript writing. All authors reviewed and approved the final version of the manuscript.

\section{Competing interests}

The authors declare no competing interests.

\section{Additional information}

Correspondence and requests for materials should be addressed to L.L. or H.C.

Reprints and permissions information is available at www.nature.com/reprints.

Publisher's note Springer Nature remains neutral with regard to jurisdictional claims in published maps and institutional affiliations.

(c) (i) Open Access This article is licensed under a Creative Commons Attribution 4.0 International cc) License, which permits use, sharing, adaptation, distribution and reproduction in any medium or format, as long as you give appropriate credit to the original author(s) and the source, provide a link to the Creative Commons license, and indicate if changes were made. The images or other third party material in this article are included in the article's Creative Commons license, unless indicated otherwise in a credit line to the material. If material is not included in the article's Creative Commons license and your intended use is not permitted by statutory regulation or exceeds the permitted use, you will need to obtain permission directly from the copyright holder. To view a copy of this license, visit http://creativecommons.org/licenses/by/4.0/.

(C) The Author(s) 2020 